\title{
Production of eicosapentaenoic acid by application of a delta- 6 desaturase with the highest ALA catalytic activity in algae
}

\author{
Haisu Shi, Xue Luo, Rina Wu* and Xiqing Yue
}

\begin{abstract}
:
Dunaliella salina is a unicellular green alga with a high a-linolenic acid (ALA) level, but a low eicosapentaenoic acid (EPA) level. In a previous analysis of the catalytic activity of delta 6 fatty acid desaturase (FADS6) from various species, FADS6 from Thalassiosira pseudonana (TpFADS6), a marine diatom, showed the highest catalytic activity for ALA. In this study, to enhance EPA production in D. salina, FADS6 from D. salina (DsFADS6) was identified, and substrate specificities for DsFADS6 and TpFADS6 were characterized. Furthermore, a plasmid harboring the TpFADS6 gene was constructed and overexpressed in D. salina. Our results revealed that EPA production reached $21.3 \pm 1.5 \mathrm{mg} / \mathrm{L}$ in $D$. salina transformants. To further increase EPA production, myoinositol (MI) was used as a growth-promoting agent; it increased the dry cell weight of $D$. salina transformants, and EPA production reached $91.3 \pm 11.6 \mathrm{mg} / \mathrm{L}$. The combination of $12 \% \mathrm{CO}_{2}$ aeration with glucose $/ \mathrm{KNO}_{3}$ in the medium improved EPA production to $192.9 \pm 25.7 \mathrm{mg} / \mathrm{L}$ in the Ds-TpFADS6 transformant. We confirmed that the increase in ALA was optimal at $8{ }^{\circ} \mathrm{C}$; the EPA percentage reached $41.12 \pm 4.78 \%$. The EPA yield was further increased to $554.3 \pm 95.6 \mathrm{mg} / \mathrm{L}$ by supplementation with $4 \mathrm{~g} / \mathrm{L}$ perilla seed meal (PeSM), $500 \mathrm{mg} / \mathrm{L} \mathrm{Ml}$, and $12 \% \mathrm{CO}_{2}$ aeration with glucose/ $\mathrm{KNO}_{3}$ at varying temperatures. EPA production and the percentage of EPA in D. salina were 343.8-fold and 25-fold higher than those in wild-type D. salina, respectively.

Importance: FADS6 from Thalassiosira pseudonana, which demonstrates high catalytic activity toward a-linolenic acid, was used to enhance EPA production by Dunaliella salina. Transformation of FADS6 from Thalassiosira pseudonana into Dunaliella salina with myoinositol, $\mathrm{CO}_{2}$, low temperatures, and perilla seed meal supplementation substantially increased EPA production in Dunaliella salina to $554.3 \pm 95.6 \mathrm{mg} / \mathrm{L}$. Accordingly, D. salina could be a potential alternative source of EPA and is suitable for its large-scale production.
\end{abstract}

Keywords: Delta 6 fatty acid desaturase, Eicosapentaenoic acid, Perilla seed meal

\section{Background}

Recent clinical and epidemiological studies have indicated that polyunsaturated fatty acids (PUFAs), such as eicosapentaenoic acid (EPA, 20:5 $5^{\Delta 5,8}, 11,14,17$ ), are essential nutrients and play crucial roles in the treatment of various human diseases [1-3], such as neuropsychiatric disorders [4], rheumatoid arthritis [5], inflammatory diseases [6, 7], hypertension [8], and cardiovascular diseases [9]. Currently, marine fish oil is the richest source of EPA; however, the depletion of global fisheries, high cost of

*Correspondence: wrn6956@163.com; yxqsyau@126.com College of Food Science, Shenyang Agricultural University, Shenyang 110866, People's Republic of China
EPA purification, and pollution of the marine environment [10] have prompted a search for alternative sources. Microorganisms, including microalgae, fungi, and bacteria, are the primary producers of EPA; microalgae are the most abundant source of EPA. Many recent studies have developed technologies to accumulate EPA directly from various microalgae [11].

Among Chlorophyceae, Dunaliella salina (D. salina) is a unicellular wall-less alga that produces $\beta$-carotene and has a high tolerance to salt, temperature and light. In addition, this alga is quite easy to cultivate and has a relatively high lipid content, especially $\alpha$-linolenic acid (ALA, 18:3 $3^{\triangle 9,12,15}$ ). However, EPA production in D. salina 
is limited. Most algae produce EPA via the $\omega-3$ pathway (Fig. 1); linoleic acid [LA, 18:2 $2^{\Delta 9,12}$ ] forms a double bond between the carbon 15 and 16 from its carboxyl end to synthesize ALA, and EPA is produced by further desaturation and elongation of the carbon chain from ALA. In this pathway, the metabolic flux of $D$. salina PUFAs is blocked, maintaining ALA production. If this flux is unblocked, the EPA level in D. salina is expected to be enhanced. This can be achieved by the transformation of an exogenous ALA-preferring delta 6 fatty acid desaturase (FADS6) gene. Based on previous analysis of the substrate specificity of FADS6 from various species for LA and ALA [12], FADS6 of Thalassiosira pseudonana (TpFADS6), a marine diatom, has higher catalytic activity toward the $\omega 3$ substrate (ALA) than that of other algae, plants, and fungi. Its conversion efficiency is $68 \%$ in the $\omega-6$ pool and $80 \%$ in the $\omega-3$ pool [13], which is the highest ALA catalytic activity by far. Accordingly, the TpFADS6 gene was transformed into $D$. salina in the present study.

Furthermore, we investigated various factors influencing EPA production in $D$. salina. Inositols are potentially valuable supplements for microalgal growth, especially myoinositol (MI) [14, 15]. Carbon sources provide energy necessary for algal growth. D. salina can use $\mathrm{CO}_{2}$ as a carbon source under photoautotrophic conditions [16]. D. salina is also capable of utilizing organic carbon sources, such as perilla seed meal (PeSM), which may promote its growth and/or EPA production [17]. PeSM solubility reaches $80 \%$ at $\mathrm{pH} 8.0$, and it contains comprehensive essential amino acids and has a high ALA content; accordingly, it is a potential substrate for EPA production in D. salina.

In this study, we identified the DsFADS6 gene from $D$. salina and characterized the substrate specificities for DsFADS6 and TpFADS6. Additionally, each FADS6 was overexpressed separately in D. salina. Furthermore, transgenic algae expressing each transgene were grown in Ben-Amotz medium to examine EPA production. Finally, we investigated the factors that influence EPA production by $D$. salina, including MI, $\mathrm{CO}_{2}$, low temperature, and PeSM supplementation.

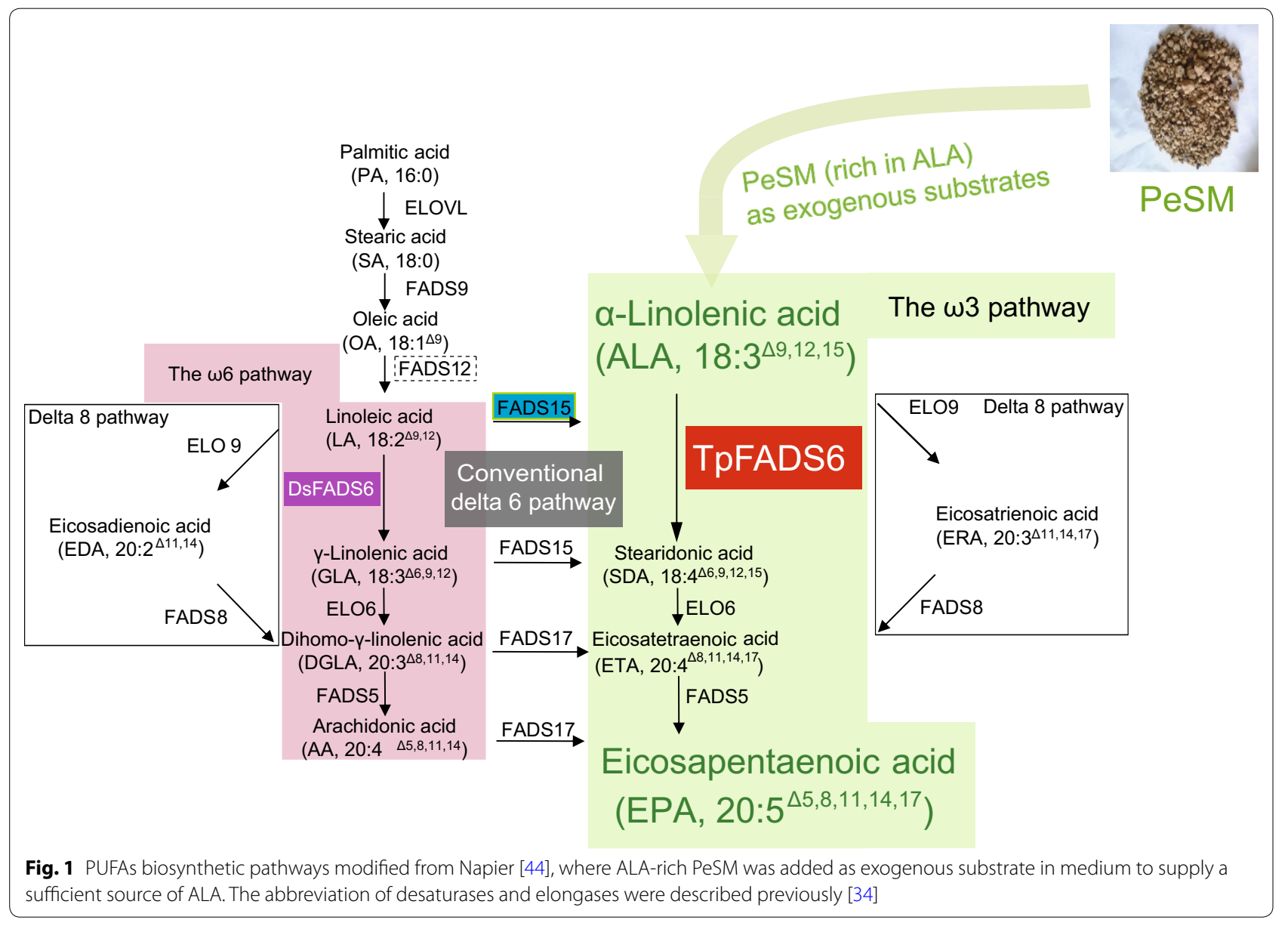




\section{Results}

\section{Identification of DsFADS6 from D. salina}

To identify genes encoding delta 6 desaturases involved in the biosynthesis of PUFAs in D. salina, a pair of degenerate primers was designed to target sequences corresponding to the heme binding motif of the cyt b5-like domain (HPGG) and the third His-rich motif (QIEHH) in DsFADS6. A 975-bp cDNA fragment from $D$. salina encoded a partial amino acid sequence containing a cyt b5-like domain in the $\mathrm{N}$ terminus and a His-rich motif in the $\mathrm{C}$ terminus; this region had high homology with delta 6 desaturases in other species.

To isolate the full-length cDNA clone, the insert was used as a probe to screen a cDNA library of $D$. salina. A full-length cDNA (DsFADS6) was identified by alignment and sequence analyses. The open reading frame of DsFADS6 was 1329 bp and it encoded 442 amino acids.

A sequence alignment indicated that DsFADS6 shares a similarity of $56.19 \%$ to FADS6 from other taxa (Fig. 2). Homology was highest near the HPGG (the cyt b5-like) domain and in the three conserved HIS-rich domains (Fig. 2). These results suggest that DsFADS6 may encode a delta 6 desaturase involved in the biosynthesis of $\gamma$-linolenic acid (GLA) and stearidonic acid (SDA) in $D$. salina.

\section{Characterization of the substrate specificity for DsFADS6 and TpFADS6}

To characterize the substrate specificity of DsFADS6 and TpFADS6, a 1329-bp fragment was amplified using FDs3 and RDs3 primers, and a 1455-bp fragment was amplified using FTp and RTp primers (primers listed in Additional file 1: Table S1). Successful expression of pYES2-DsFADS6 and pYES2-TpFADS6 was confirmed by western blotting in Saccharomyces cerevisiae. The fatty acid compositions are summarized in Table 1. When a single substrate was added, LA was catalyzed by DsFADS6 and the conversion rate was $24.3 \pm 1.3 \%$; however, ALA was not catalyzed by DsFADS6. When both substrates were added at the same time, the LA conversion rate by DsFADS6 was $23.8 \pm 1.8 \%$, and ALA was not catalyzed. These results show that DsFADS6 can catalyze LA conversion to GLA, but is not capable of catalyzing ALA.

When a single substrate was added, both substrates were catalyzed by TpFADS6 and their conversion rates

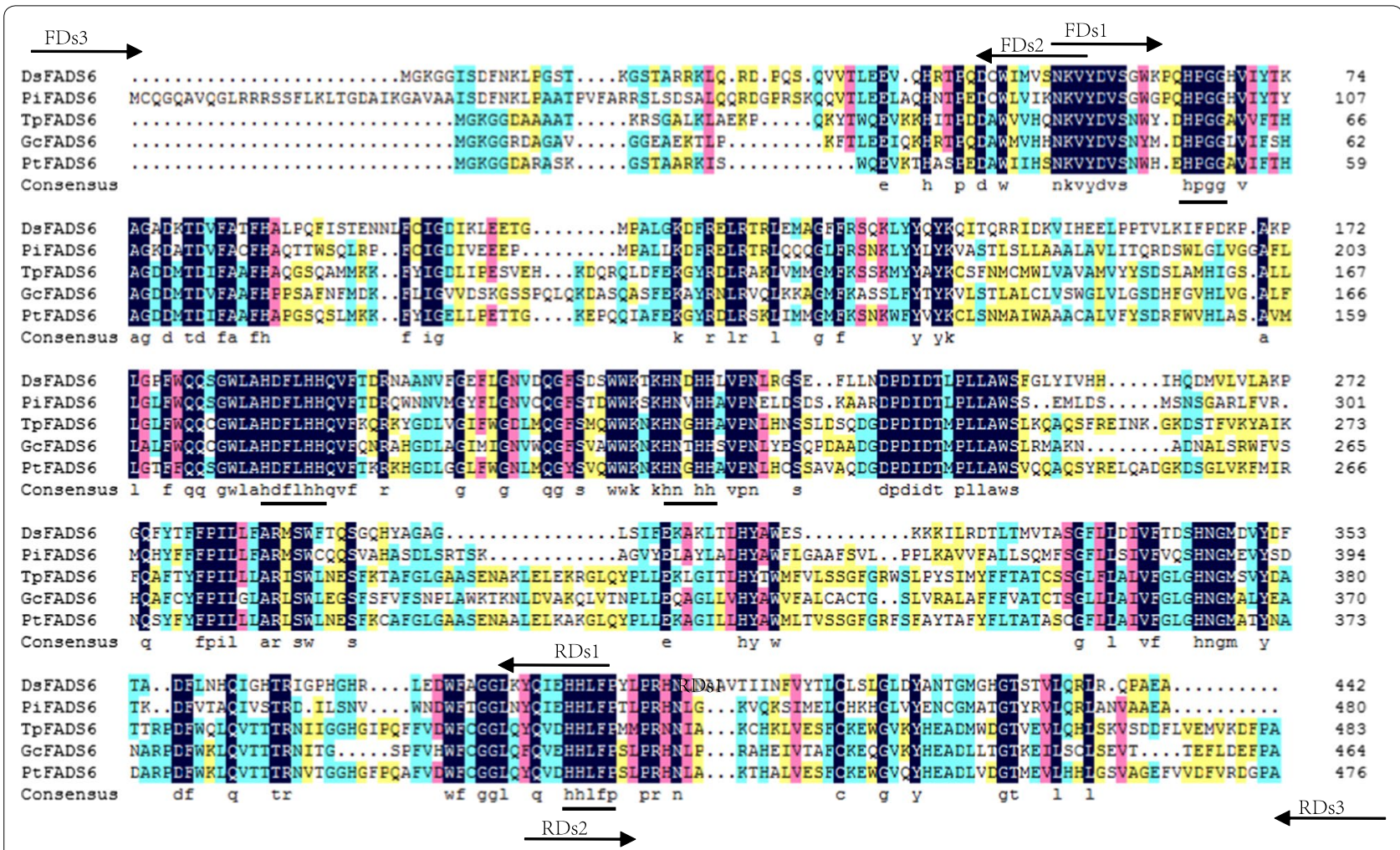

Fig. 2 Multiple Sequence alignment of deduced amino acids of DsFADS6 with that of Parietochloris incisa (PiFADS6), Thalassiosira pseudonana (TpFADS6), Glossomastix chrysoplasta (GcFADS6) and Phaeodactylum tricornutum (PtFADS6) (GenBank accession no. GU390532, AY817155, AAU11445 and AY082393) using DNAMAN. The three conserved HIS-rich motifs and a cyt b5-like domain are underlined. The arrows indicated the binding location of the degenerate primers used for amplification of partial sequence 
Table 1 Fatty acid compositions (\% w/w) of the total lipid contents of yeast transformants harboring the control plasmid (pYES2) and the recombinant plasmids (pYES2-DsFADS6 and pYES2-TpFADS6)

\begin{tabular}{|c|c|c|c|c|c|c|c|c|c|}
\hline \multirow{2}{*}{$\begin{array}{l}\text { Transformants } \\
\text { Fatty acid }\end{array}$} & \multicolumn{3}{|c|}{ pYES2 (control) } & \multicolumn{3}{|c|}{ pYES2-DsFADS6 } & \multicolumn{3}{|c|}{ pYES2-TpFADS6 } \\
\hline & $+\mathrm{LA}$ & $+A L A$ & $+\mathrm{LA}$ and +ALA & $+L A$ & $+\mathrm{ALA}$ & $+\mathrm{LA}$ and $+\mathrm{ALA}$ & $+\mathrm{LA}$ & $+A L A$ & $+\mathrm{LA}$ and +ALA \\
\hline 16:0 (PA) & $22.9 \pm 0.2$ & $25.6 \pm 0.2$ & $20.5 \pm 0.1$ & $25.5 \pm 0.4$ & $21.9 \pm 0.1$ & $22.7 \pm 0.3$ & $23.4 \pm 0.3$ & $22.0 \pm 0.6$ & $24.0 \pm 0.4$ \\
\hline $16: 1(\mathrm{PA})$ & $12.7 \pm 0.3$ & $14.6 \pm 0.2$ & $12.9 \pm 0.3$ & $14.4 \pm 0.2$ & $16.3 \pm 0.3$ & $11.8 \pm 0.2$ & $14.6 \pm 0.2$ & $14.4 \pm 0.4$ & $13.1 \pm 0.6$ \\
\hline $18: 0(\mathrm{SA})$ & $11.4 \pm 0.4$ & $10.2 \pm 0.6$ & $11.8 \pm 0.7$ & $10.9 \pm 0.4$ & $12.5 \pm 0.3$ & $10.2 \pm 0.4$ & $12.0 \pm 0.7$ & $14.3 \pm 0.5$ & $9.3 \pm 0.2$ \\
\hline 18:1 (OA) & $12.7 \pm 0.8$ & $10.3 \pm 0.7$ & $11.6 \pm 0.4$ & $12.5 \pm 0.2$ & $11.8 \pm 0.6$ & $12.6 \pm 0.4$ & $11.3 \pm 0.4$ & $11.2 \pm 0.3$ & $10.3 \pm 0.4$ \\
\hline $18: 2(L A, \omega-6)$ & $38.2 \pm 0.3$ & ND & $23.6 \pm 0.1$ & $29.6 \pm 0.6$ & ND & $16.1 \pm 0.2$ & $11.5 \pm 0.5$ & ND & $5.9 \pm 0.6$ \\
\hline $18: 3(\mathrm{ALA}, \omega-3)$ & ND & $39.6 \pm 0.5$ & $21.2 \pm 0.6$ & ND & $37.5 \pm 0.2$ & $21.6 \pm 0.3$ & ND & $4.3 \pm 0.6$ & $2.7 \pm 0.2$ \\
\hline $18: 3(G L A, \omega-6)$ & ND & $\mathrm{ND}$ & $\mathrm{ND}$ & $7.2 \pm 0.1$ & ND & $5.0 \pm 0.1$ & $27.3 \pm 0.3$ & $\mathrm{ND}$ & $13.3 \pm 0.3$ \\
\hline $18: 4(\mathrm{SDA}, \omega-3)$ & ND & ND & ND & ND & ND & ND & ND & $34.9 \pm 0.4$ & $16.7 \pm 0.2$ \\
\hline LA conversion rate $^{a}$ & - & - & - & $24.3 \pm 1.3$ & - & $23.8 \pm 1.8$ & $70.4 \pm 0.2$ & - & $69.3 \pm 0.3$ \\
\hline ALA conversion rate ${ }^{a}$ & - & - & - & - & 0 & 0 & - & $89.0 \pm 2.2$ & $86.1 \pm 1.3$ \\
\hline
\end{tabular}

ND not detected

a Conversion rate $=100 \times([$ product $] /[$ product + substrate $])$

were $70.4 \pm 0.2$ and $89.0 \pm 2.2 \%$, respectively. When both substrates were added simultaneously, the conversion rates of TpFADS6 for both substrates were similar to those observed when they were added individually, consistent with the results of Thierry et al. [13]. These results show that TpFADS6 is highly capable of catalyzing the conversion of both substrates to GLA and SDA. However, TpFADS6 did not exhibit substrate specificity.

Substrate concentration has no effect on catalytic activity in yeast expressing DsFADS6; similarly, we found that there was no substrate specificity for TpFADS6, irrespective of fatty acid concentration (data not shown).

\section{Overexpression of TpFADS6 in D. salina}

To construct expression plasmids, a 1329-bp fragment was amplified using pGEM-FDs/pGEM-RDs primers, and a 1455-bp fragment was amplified using pGEMFTp/pGEM-RTp primers. Both fragments were ligated into the pGEM-CAT vector to obtain the pGEM-CATDsFADS6 and pGEM-CAT-TpFADS6 vectors (Additional file 2: Fig. S1a, b). The resulting pGEM-CAT-DsFADS6 and pGEM-CAT-TpFADS6 vectors were electrotransformed separately into $D$. salina. After transformation, small green colonies appeared at a frequency of 10-20 colonies/plate after 15 days. Ten pGEM-CAT-DsFADS6 transformants and 10 pGEM-CAT-TpFADS6 transformants were picked and cultured in liquid medium.

PCR amplification was used to identify DsFADS6 and TpFADS6 gene fragments in the transformants (Additional file 2: Fig. S1c, d). The target gene was detected in all transformants. Based on ELISA, CAT proteins were successfully expressed in all transformants and there were no significant differences in the expression level of $C A T$ genes among D. salina transformants (Fig. 3a, b).
The expression levels of the DsFADS6 and TpFADS6 genes in all samples (including wild-type [WT] D. salina, pGEM-CAT-DsFADS6 transformants, and pGEM-CATTpFADS6 transformants) were analyzed after a 15-day cultivation period. Based on RT-qPCR, the transcript level of the DsFADS6 gene in pGEM-CAT-DsFADS6 transformants was approximately two to ninefold higher than that in WT D. salina (Fig. 3c). The No. 9 pGEMCAT-DsFADS6 transformant (Ds-DsFADS6-9) was used as a negative control; it had the highest transcript level of DsFADS6 (9.4-fold higher than that in WT D. salina). There were significant differences in the transcript levels of TpFADS6 among pGEM-CAT-TpFADS6 transformants. The No. 4 pGEM-CAT-TpFADS6 transformant (Ds-TpFADS6-4 transformant) had the highest TpFADS6 transcript level (8.5-fold higher than that in WT $D$. salina), followed by the Nos. 8 and 10 pGEM-CATTpFADS6 transformants (7.1- and 7.6-fold higher than that in WT D. salina, respectively), and the transcript level was lower for the other pGEM-CAT-TpFADS6 transformants (Fig. 3d). The fatty acid profiles of DsTpFADS6-4, Ds-TpFADS6-8, and Ds-TpFADS6-10 were examined.

\section{Fatty acid profile of transformants}

All transformants and WT D. salina were grown in Ben-Amotz medium at $26^{\circ} \mathrm{C}$ for 30 days, and their fatty acid profiles were determined. There were no significant differences in dry cell weight, total fatty acid content, and C16 series levels among these transformants (Table 2). However, ALA levels were low in Ds-TpFADS6 transformants, in which the LA levels decreased to approximately $2 \mathrm{mg} / \mathrm{L}$, and the EPA levels increased to $21.3 \pm 1.5 \mathrm{mg} / \mathrm{L}$, compared to $1.6 \mathrm{mg} / \mathrm{L}$ in WT D. salina 

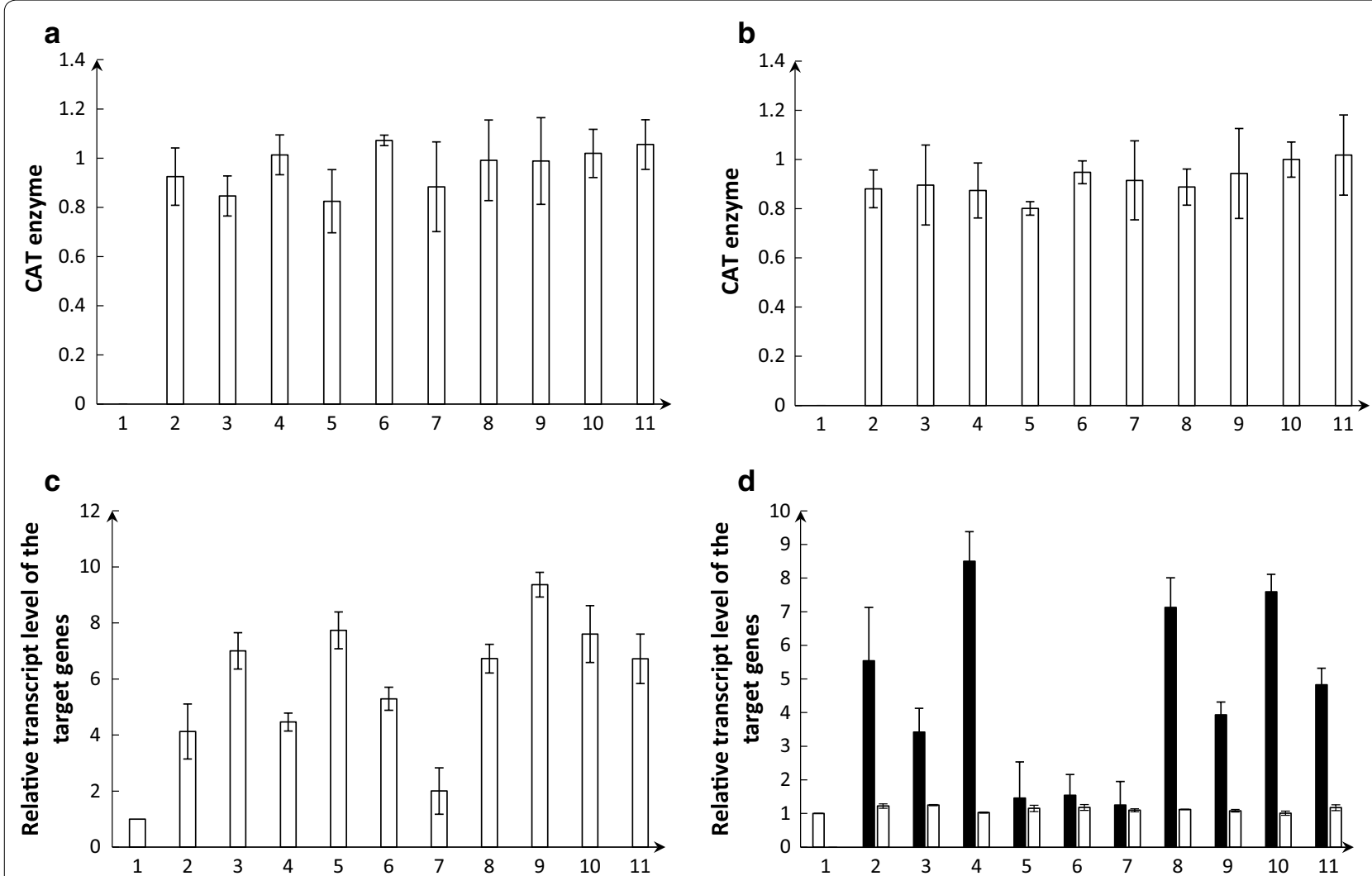

Fig. 3 Analysis of CAT enzyme of pGEM-CAT-DsFADS6 transformants and pGEM-CAT-TpFADS6 transformants, respectively. Ten pGEM-CAT-DsFADS6 transformants and ten pGEM-CAT-TpFADS6 transformants were randomly picked out, and CAT enzymes were analyzed by ELISA method. Each column represented individual CAT protein of each transformants we picked out. a pGEM-CAT-DsFADS6 transformants; b pGEM-CAT-TpFADS6 transformants. Relative transcript level of DsFADS6, TpFADS6 in pGEM-CAT-DsFADS6 (c) and pGEM-CAT-TpFADS6 (d) transformants and the control strain (wild-type D. salina). The open bars represented the DsFADS6 transcript level and the black bars represented the TpFADS6 transcript level. Relative transcript level of the control strain was defined as 1

Table 2 Principal fatty acid profiles of each D. salina transformant

\begin{tabular}{lllllllc}
\hline & DCW $(\mathbf{m g} / \mathbf{L})$ & TFA $(\mathbf{m g} / \mathbf{L})$ & C16 series $_{(\mathbf{m g} / \mathbf{L})}$ & LA $(\mathbf{m g} / \mathbf{L})$ & ALA $(\mathbf{m g} / \mathbf{L})$ & EPA (mg/L) & The percentage of EPA (TFA \%) \\
\hline WT D. salina & $266.0 \pm 30.7$ & $78.6 \pm 1.6$ & $41.2 \pm 1.5$ & $5.3 \pm 0.2$ & $26.6 \pm 4.7$ & $1.6 \pm 0.2$ & $1.91 \pm 0.21$ \\
Ds-DsFADS6-9 & $273.4 \pm 22.0$ & $73.2 \pm 1.6$ & $38.9 \pm 2.6$ & $5.4 \pm 0.3$ & $27.3 \pm 2.5$ & $1.5 \pm 0.1$ & $2.04 \pm 0.14$ \\
Ds-TpFADS6-4 & $261.8 \pm 18.2$ & $74.1 \pm 4.9$ & $37.6 \pm 1.9$ & $2.0 \pm 0.4$ & $2.6 \pm 0.6$ & $20.7 \pm 3$ & $28.12 \pm 5.75$ \\
Ds-TpFADS6-8 & $239.0 \pm 33.5$ & $79.9 \pm 5.5$ & $35.5 \pm 2.6$ & $2.1 \pm 0.2$ & $2.8 \pm 0.2$ & $21.3 \pm 1.5$ & $26.72 \pm 2.26$ \\
Ds-TpFADS6-10 & $274.5 \pm 19.4$ & $83.8 \pm 3.3$ & $38.9 \pm 2.1$ & $2.2 \pm 0.2$ & $3.3 \pm 0$ & $19.1 \pm 2.3$ & $22.9 \pm 3.65$ \\
\hline
\end{tabular}

a C16 series included 16:0, 16:1, 16:2, 16:3 and 16:4

(Table 2). The EPA content (expressed as a \% of TFA) was $28.12 \pm 5.75 \%$ in the Ds-TpFADS6- 4 transformant, compared to $1.91 \pm 0.21 \%$ in WT D. salina. (Table 2).

\section{Biomass-enhancing effect of $\mathrm{MI}$ supplementation}

Although TpFADS6 can promote the conversion of ALA to EPA in Ds-TpFADS6 transformants, the biomass of transformants was still low, limiting large-scale production. We attempted to resolve this problem by supplementing cultures with MI to stimulate the growth of transformants. Kichul et al. showed that supplementation with $500 \mathrm{mg} / \mathrm{L} \mathrm{MI}$ had the greatest growth-promoting effect on $D$. salina (1.34 times higher growth than that of the control) [15].

The effects of MI on the growth of each transformant are summarized in Fig. 4. The dry cell weights (DCW) of 

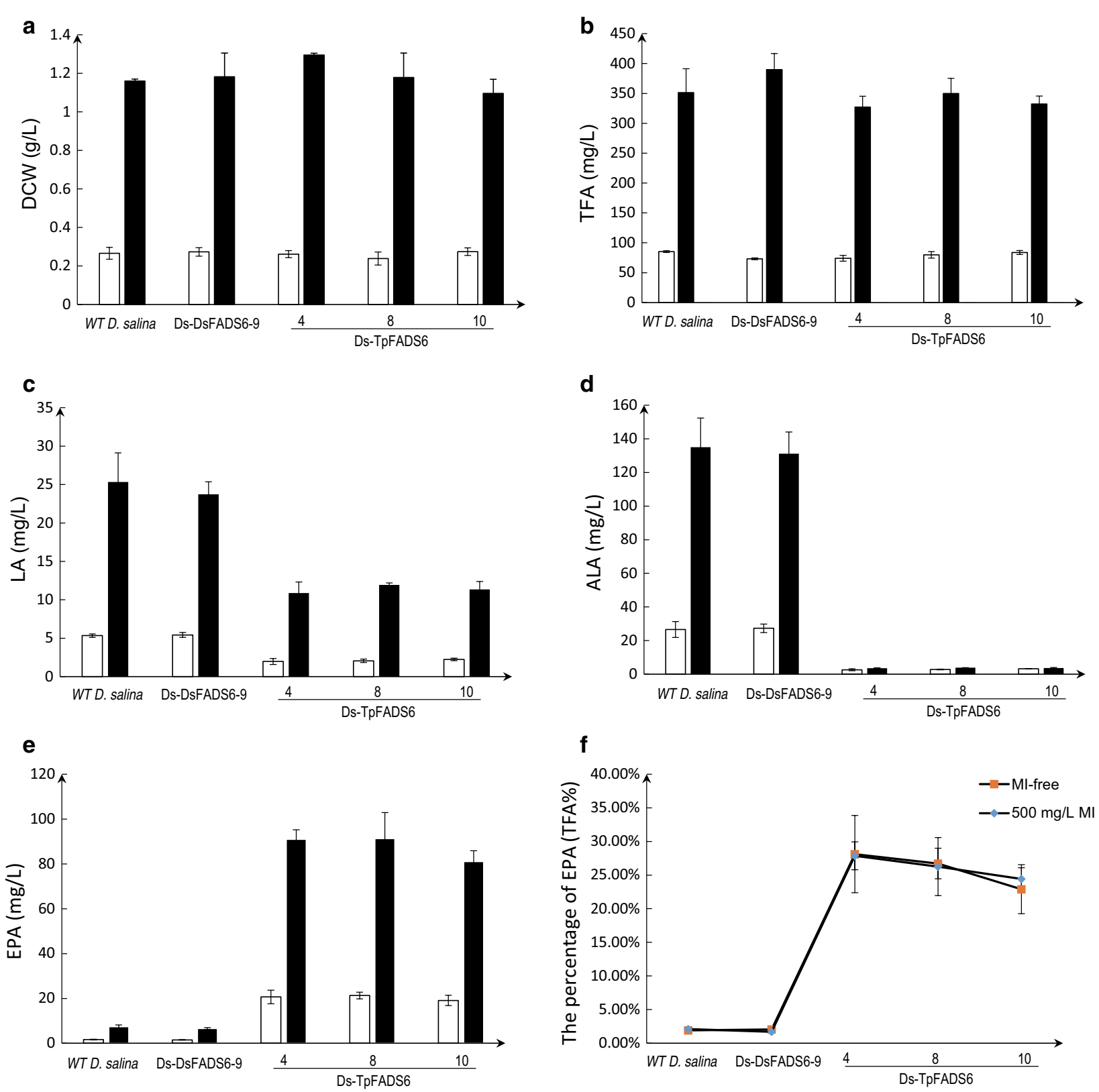

-MI-free $\cdot 500 \mathrm{mg} / \mathrm{L} \mathrm{MI}$

Fig. 4 The effect of MI on each alga growth and principal fatty acid profiles. DCW (dry cell weight) (a), TFA (total fatty acids) (b), LA level (c), ALA level (d), EPA level (e) and the percentage of EPA (f) were determined in the control strain (WT D. salina) and transformants (including Ds-DsFADS6-9, Ds-DsFADS6-4, Ds-TpFADS6-8, and Ds-TpFADS6-10). Each alga was cultured in Ben-Amotz medium with 500 mg/L Ml

WT D. salina and the transformants was increased significantly by supplementation with $500 \mathrm{mg} / \mathrm{L} \mathrm{MI}$, and reached sixfold higher than those of the MI-free control (Fig. 4a); TFA levels also increased (Fig. 4b). However, no significant changes were observed in the percentage of TFA (DCW \%) among strains after the addition of MI. The levels of LA and ALA in Ds-TpFADS6 transformants was decreased by supplementation with MI, similar to the levels of the MI-free control (Fig. 4c, d). The EPA yield and percentage of EPA (TFA \%) in the three Ds-TpFADS6 transformants were increased to $91.0 \pm 4.2 \mathrm{mg} / \mathrm{L}$ (27.9 $\pm 2.1 \%), 91.3 \pm 11.6 \mathrm{mg} / \mathrm{L}(26.3 \pm 4.3 \%)$, and $81.1 \pm 4.8 \mathrm{mg} / \mathrm{L}(24.4 \pm 1.7 \%)$ by supplementation with $500 \mathrm{mg} / \mathrm{L}$ MI (Fig. 4e). However, there were no significant 
changes in the percentage of EPA (TFA \%) between the MI group and the MI-free control (Fig. 4f).

\section{Lipid enhancement by supplementation with glucose/ $\mathrm{KNO}_{3}$ and $\mathrm{CO}_{2}$}

Glucose and $\mathrm{CO}_{2}$ in the culture medium can be used as carbon sources for algal growth, and their levels have significant effects on lipid accumulation in D. salina $[16,18]$. We combined $\mathrm{CO}_{2}$ with glucose $/ \mathrm{KNO}_{3}$ and examined DCW and lipid accumulation in D. salina transformants.

Our results revealed that the DCW of WT D. salina and the transformants was increased significantly by aeration of $9 \% \mathrm{CO}_{2}$, to approximately sevenfold higher than those of the control and approximately $50 \%$ greater than those of the MI group. The DCW of WT D. salina and all transformants was decreased by aeration with $12 \% \mathrm{CO}_{2}$ (Fig. 5a). However, the TFA of all algae after aeration with $12 \% \mathrm{CO}_{2}$ was greater than that observed after aeration with $9 \% \mathrm{CO}_{2}$ (Fig. 5b). Based on the curve of the percentage of TFA (DCW \%), the TFA content in WT D. salina and all of the transformants was increased by approximately twofold by aeration with $12 \% \mathrm{CO}_{2}$ compared to that with $9 \% \mathrm{CO}_{2}$ and the control (Fig. 5c). The levels of LA and ALA in all algae exhibited similar patterns to those of MI-supplemented cultures (Fig. 5d, e). The DsTpFADS6 transformants presented noticeably higher EPA production with $9 \%$ and $12 \% \mathrm{CO}_{2}$ aeration, reaching approximately $122.6 \pm 7.8$ and $192.9 \pm 25.7 \mathrm{mg} / \mathrm{L}$, respectively (Fig. 5f). However, there were no significant differences in the percentage of EPA (\%TFA) (Fig. 5g). These results confirm that $\mathrm{CO}_{2}$ aeration in medium can enhance biomass and lipid production in D. salina; under $12 \% \mathrm{CO}_{2}$ aeration, the level of EPA in Ds-TpFADS6 transformants increased by more than 19.2 fold relative to $D$. salina.

\section{Promoting the conversion of LA to ALA}

The above results indicated that most ALA was converted to EPA, and only a small quantity remained in Ds-TpFADS6 transformants. We determined the fatty acid profile of transformants after they were cultured in Ben-Amotz medium by supplementation with glucose/ $\mathrm{KNO}_{3}$ and $12 \% \mathrm{CO}_{2}$ aeration at $4{ }^{\circ} \mathrm{C}$ for 5 days. Unexpectedly, ALA levels increased and LA levels decreased (Fig. 6a, b). A potential explanation for this result was that the DsFADS15 enzyme had greater catalytic activity at a low temperature, promoting the conversion of LA to ALA. However, the EPA levels did not change significantly, suggesting that the DsFADS6 enzyme had lower catalytic activity at a low temperature than at $26{ }^{\circ} \mathrm{C}$. We examined a temperature gradient $\left[4,8,12,16\right.$, and $26^{\circ} \mathrm{C}$ (control)] to determine the optimal conditions for ALA accumulation. The LA level at $8{ }^{\circ} \mathrm{C}$ was the lowest among the four temperatures 5 days after a 30-day cultivation period (Fig. 6c), and the ALA level at $8{ }^{\circ} \mathrm{C}$ was the highest among all temperatures (Fig. 6d). These results suggested that the DsFADS15 enzyme had the highest catalytic activity at $8{ }^{\circ} \mathrm{C}$. After optimization of the culture temperature, each transformant was cultured for an additional 5, 10,15 , or 20 days after 30 days of growth at $8{ }^{\circ} \mathrm{C}$. The LA level decreased and the ALA level increased as the cultivation progressed (Fig. 6e, f). The DCW, TFA, and EPA levels in this condition (at $8{ }^{\circ} \mathrm{C}$ and for another 20 days) did not exhibit significant changes (data not shown).

By analyzing the growth curve for one of the DsTpFADS6 transformants (Ds-TpFADS6-8), we determined its DCW, TFA, ALA, and EPA levels for 30-day cultivation at $26{ }^{\circ} \mathrm{C}$. There were no significant differences in DCW and TFA between two samples during the whole cultivation period (Fig. 7a, b), whereas the LA levels increased as the cultivation period progressed, with a greater increase from days 15 to 20 (Fig. 7c). The LA level showed a gradual increase.

To maximize the ALA level, we maintained the culture temperature at the optimal temperature $\left(8{ }^{\circ} \mathrm{C}\right)$ from 20 to 25 days. The DCW and TFA levels at varying temperatures decreased slightly relative to those in a constant temperature (Fig. 7a, b). LA levels decreased sharply between 20 and 25 days, from $16.1 \pm 1.7$ to $7.3 \pm 0.8 \mathrm{mg} / \mathrm{L}$ in WT D. salina (from $7.0 \pm 0.8$ to $2.1 \pm 0.3 \mathrm{mg} / \mathrm{L}$ in the Ds-TpFADS6-8 transformant) (Fig. 7c). By contrast, ALA levels increased rapidly during this time period, from $115.6 \pm 11.8$ to $233.1 \pm 24.1 \mathrm{mg} / \mathrm{L}$ in WT D. salina (from $6.4 \pm 0.6$ to $24.7 \pm 2.1 \mathrm{mg} / \mathrm{L}$ in the Ds-TpFADS6-8 transformant) (Fig. 7d). We confirmed that the DsFADS15 enzyme presented high catalytic activity at $8{ }^{\circ} \mathrm{C}$. The EPA level in the Ds-TpFADS6-8 transformant reached $216.8 \pm 22.6 \mathrm{mg} / \mathrm{L}$, which was only $24 \mathrm{mg} / \mathrm{L}$ higher than the yield obtained at a constant temperature (Fig. 7e). However, the percentage of EPA (TFA $\%$ ) increased to $41.12 \pm 4.78 \%$ at the end of cultivation, which was 1.5-fold higher than that observed at a constant temperature (27.64 $\pm 1.30 \%)$ (Fig. $7 f)$.

\section{EPA enhancement by PeSM supplementation}

To further increase EPA production in D. salina, PeSM was added to the cultures. PeSM was composed of approximately 1\% ALA (Additional file 1: Table S2). The DCW of WT D. salina and all of the transformants was increased significantly by supplementation with 1 , 2 , and $4 \mathrm{~g} / \mathrm{L}$ PeSM, up to 2.6 -fold $(2.6 \pm 0.2 \mathrm{~g} / \mathrm{L}$ in DsTpFADS6-10 with $4 \mathrm{~g} / \mathrm{L}$ PeSM) higher than that of the PeSM-free control $(1.0 \pm 0.1 \mathrm{~g} / \mathrm{L}$ in Ds-TpFADS6-10) (Fig. 8a). The levels of TFA, LA, ALA, and EPA also increased (Fig. 8b-e). The EPA yield in the DsTpFADS6-10 transformant was increased to $306.5 \pm 57.9$, 

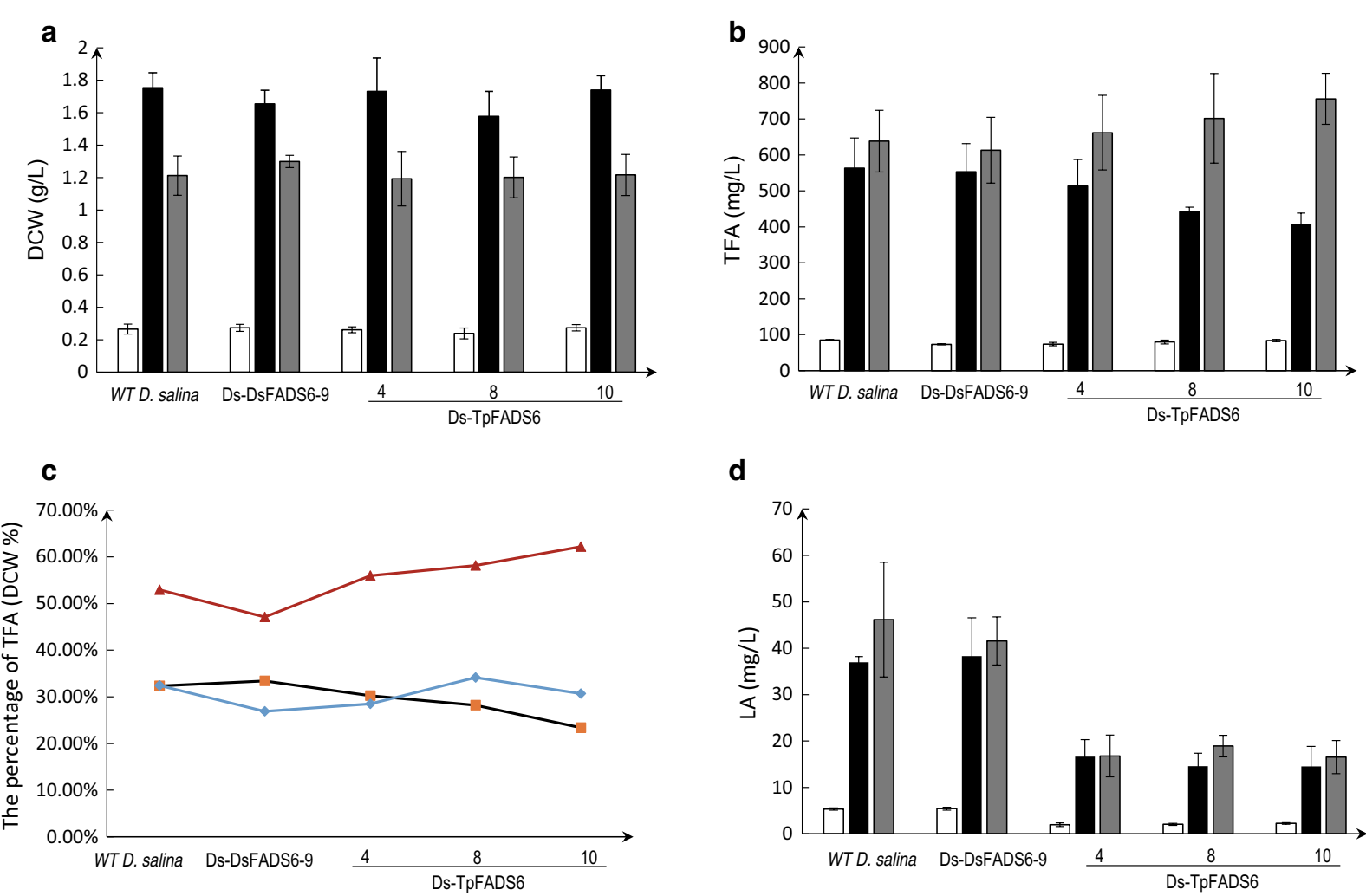

d
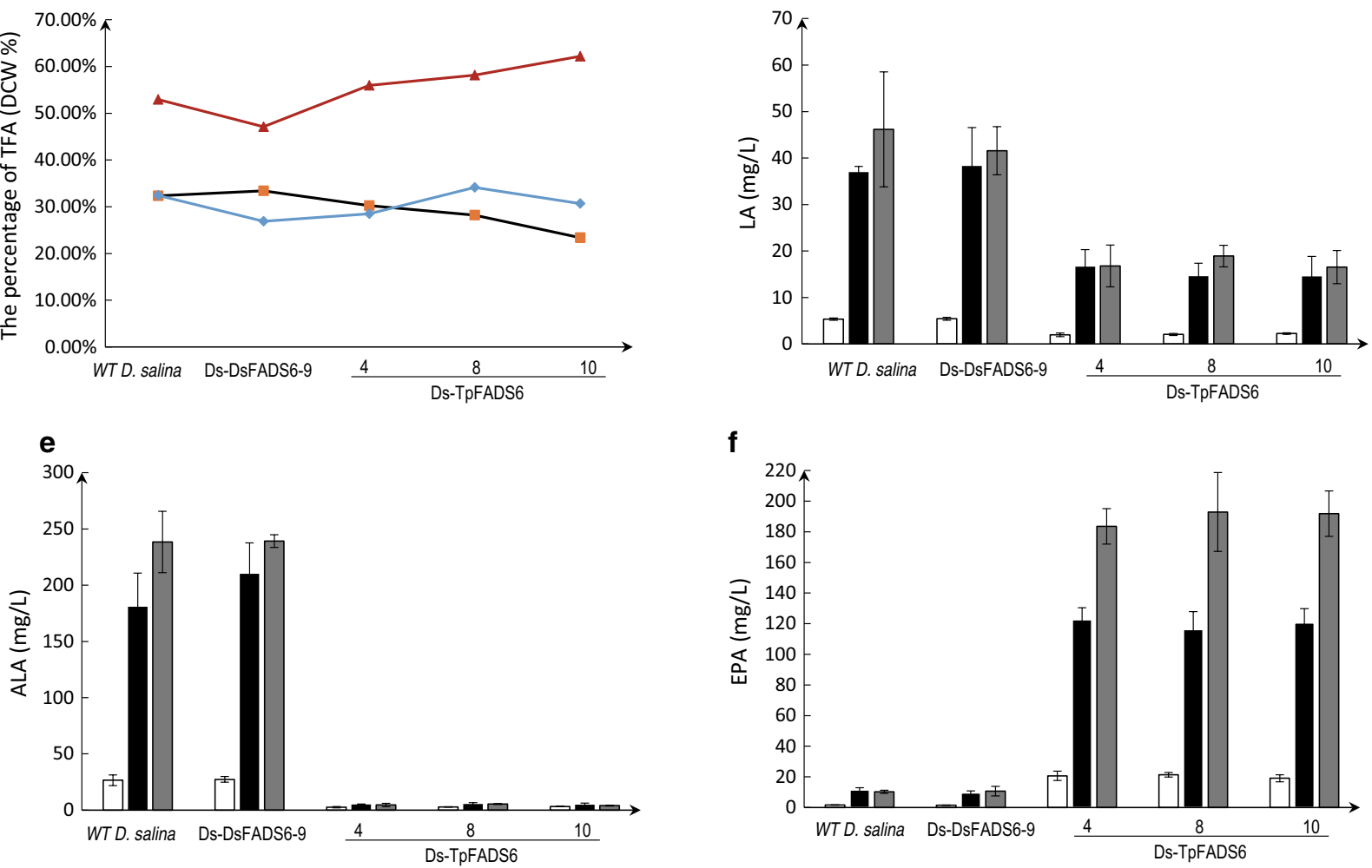

f
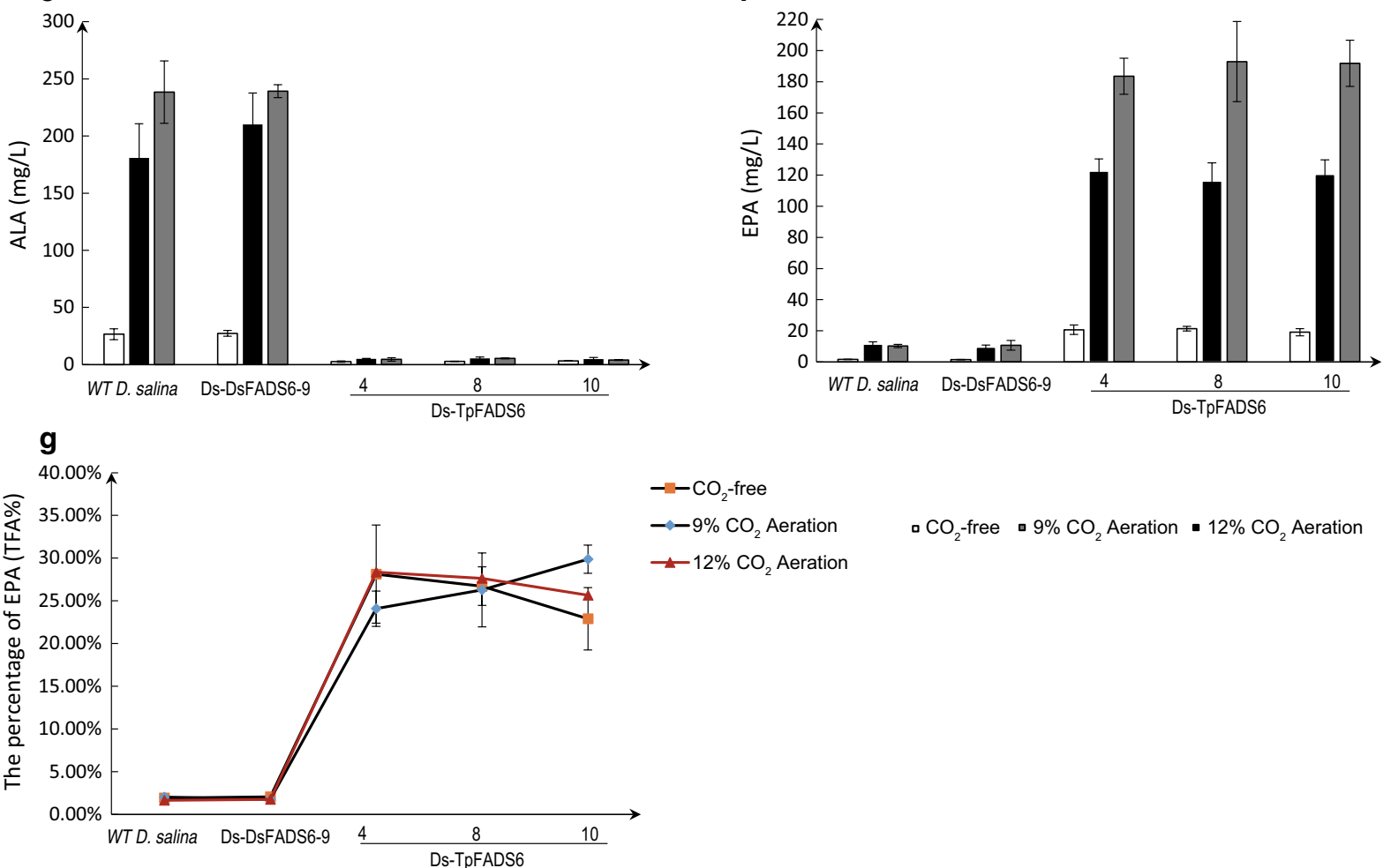

Fig. 5 The effect of $\mathrm{CO}_{2}$ on each alga growth and principal fatty acid profiles. DCW (a), TFA (b), the percentage of TFA (DCW \%) (c), LA level (d), ALA level (e), EPA level (f) and the percentage of EPA (TFA\%) (g) were determined in the control strain (WT D. salina) and transformants (including DsDsFADS6-9, Ds-DsFADS6-4, Ds-TpFADS6-8, and Ds-TpFADS6-10). Each alga was cultured in Ben-Amotz medium with $500 \mathrm{mg} / \mathrm{L} \mathrm{Ml}_{\text {and }} 9$ or $12 \% \mathrm{CO}_{2}$ aeration 


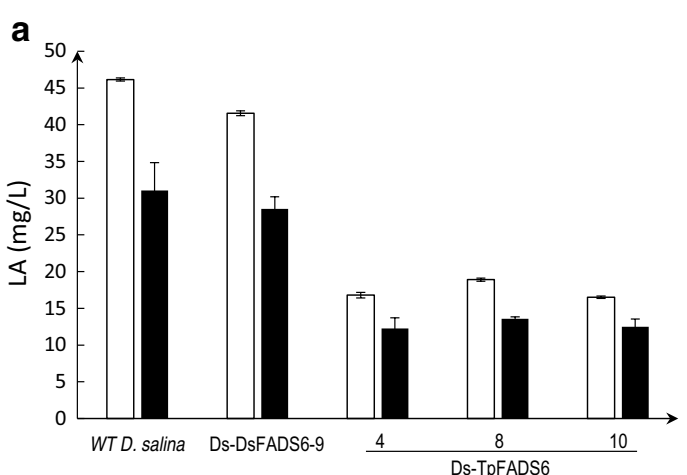

C
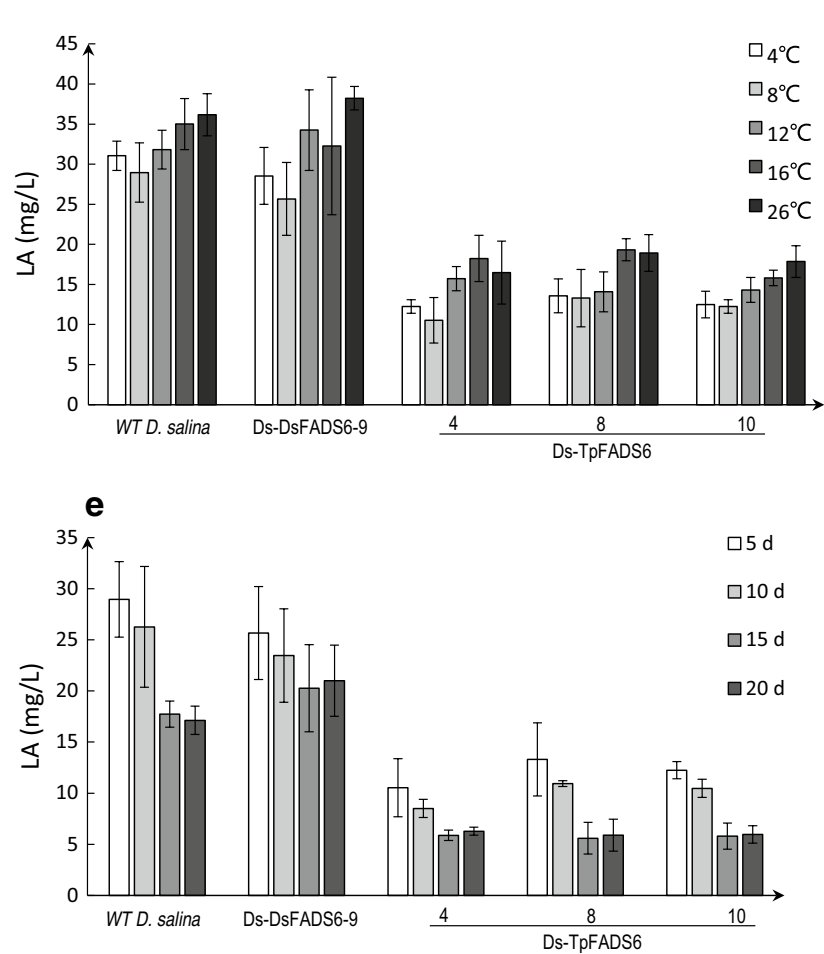

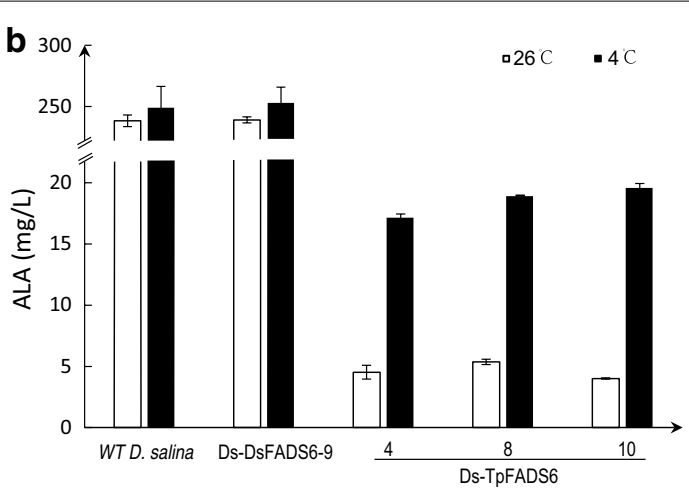

d

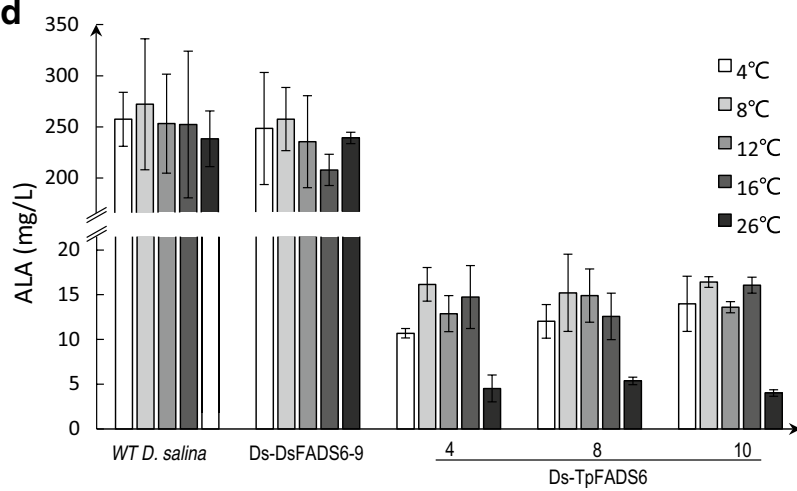

$\mathbf{f}$

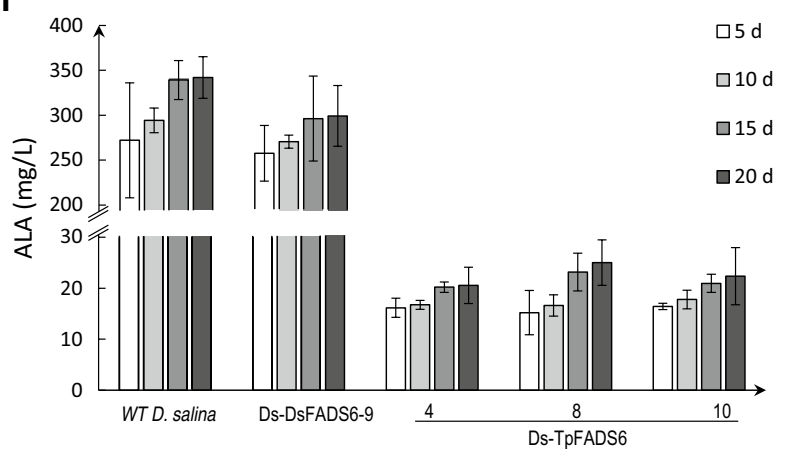

Fig. 6 The effect of low temperature on promoting the conversion of LA to ALA. LA level (a) and ALA level (b) were determined in the control strain (WT D. salina) and transformants (including Ds-DsFADS6-9, Ds-DsFADS6-4, Ds-TpFADS6-8, and Ds-TpFADS6-10). Each alga was cultured in Ben-Amotz medium by supplementation with $500 \mathrm{mg} / \mathrm{L} \mathrm{Ml}$, glucose/ $/ \mathrm{KNO}_{3}$ and $12 \% \mathrm{CO}_{2}$ aeration and conserved by $4{ }^{\circ} \mathrm{C}$ for 5 days. Optimization of culture temperature and time for each alga. LA level (c) and ALA level (d) were determined in the control strain (WT D. salina) and transformants (including Ds-DsFADS6-9, Ds-DsFADS6-4, Ds-TpFADS6-8, and Ds-TpFADS6-10) under 4, 8, 12, 16, and $26^{\circ} \mathrm{C}$ (control) after 30-day cultivation for 5 days. After optimization of culture temperature, each transformant was cultured for another 5, 10, 15 or 20 days after 30-day growth under the optimal temperature. Each alga was cultured in Ben-Amotz medium by supplementation with $500 \mathrm{mg} / \mathrm{L} \mathrm{Ml}$, glucose/ $/ \mathrm{KNO}_{3}$ and $12 \% \mathrm{CO}_{2}$ aeration. $\mathrm{LA}$ level (e) and ALA level (f) were determined in the control strain (WT D. salina) and transformants (including Ds-DsFADS6-9, Ds-DsFADS6-4, Ds-TpFADS6-8, and Ds-TpFADS6-10) under optimal temperature

$340.2 \pm 50.0$, and $554.3 \pm 95.6 \mathrm{mg} / \mathrm{L}$ by supplementation with 1, 2, and $4 \mathrm{~g} / \mathrm{L}$ PeSM, respectively (Fig. 8e). LA levels were reduced by half, and only small amounts of ALA were detected. In addition, the Ds-TpFADS6-10 transformant accumulated EPA to a content of $47.81 \pm 7.42 \%$ of the TFA after supplementation with $2 \mathrm{~g} / \mathrm{L}$ PeSM (Fig. 8f, Additional file 3: Fig. S2).

\section{Discussion}

Most algae synthesize VLC-PUFA via the FADS6 pathway (Fig. 1) [19]. Although D. salina is rich in ALA, the substrate specificity of DsFADS6 was unclear. We confirmed that it only catalyzed LA, and its conversion rate was low $(24.3 \pm 1.3 \%)$. In addition, TpFADS6 had no substrate specificity for the two substrates LA and ALA, but had 


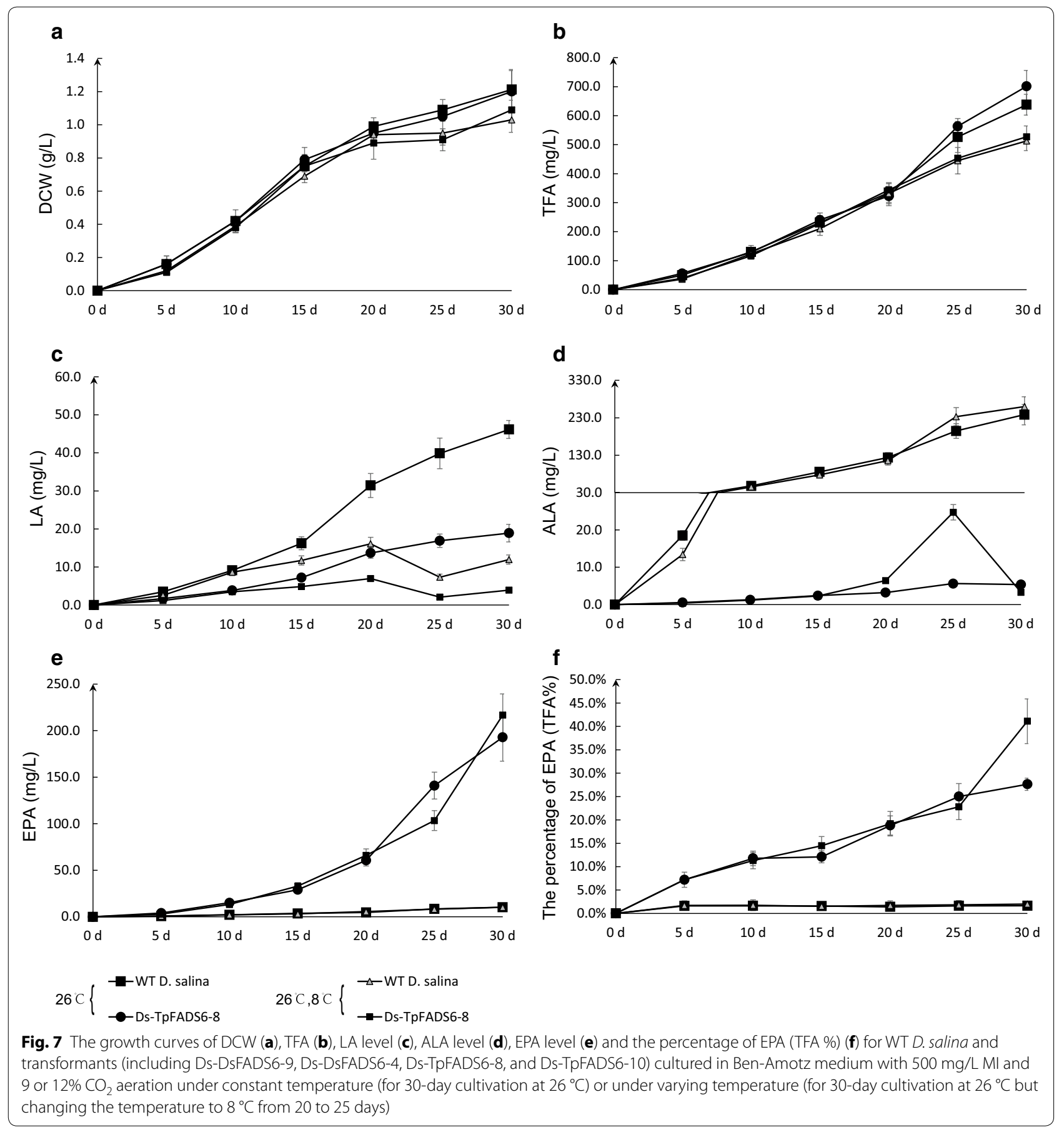

a much higher ability to catalyze ALA and LA relative to that of FADS6 in Micromonas pusilla [12]. Therefore, we reasoned that transformation of the TpFADS6 gene into $D$. salina may promote $\omega 3$-PUFA accumulation.

Cho et al. reported that exogenous genes can be easily introduced into $D$. salina [20]. We achieved the successful transformation of $D$. salina with the DsFADS6 and TpFADS6 genes using the electroporation method.
However, the expression levels of the genes varied among transformants. This variation has two potential explanations: the DsFADS6 or TpFADS6 gene was randomly inserted into the $D$. salina genome at one or more sites, or the integration sites were random.

Both LA and ALA levels decreased in TpFADS6 transformants, and ALA levels decreased more substantially. In contrast, EPA levels and the percentage of EPA (TFA 

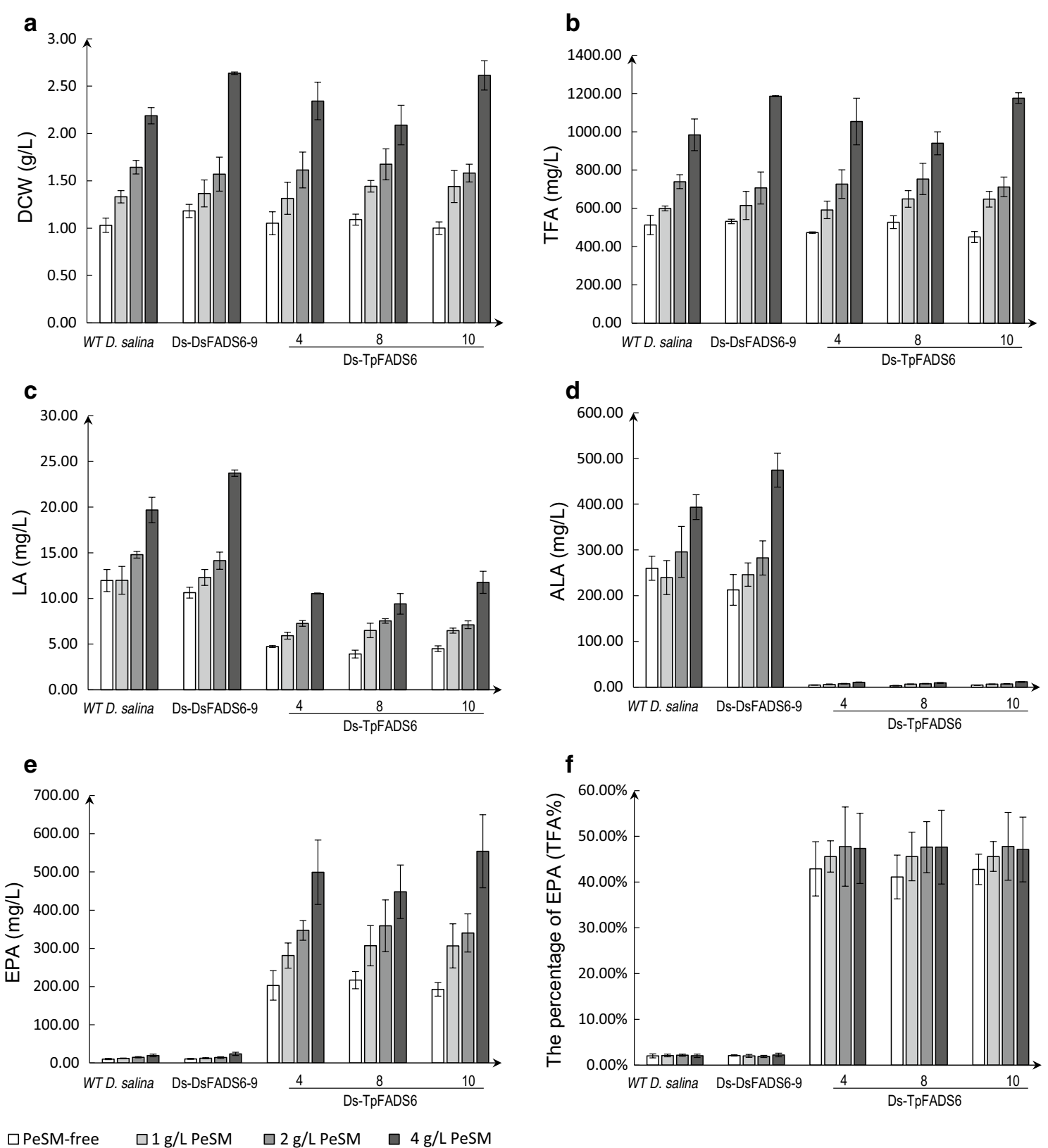

Fig. 8 The effect of different concentrations of PeSM on each alga growth and principal fatty acid profiles. DCW (a), TFA (b), LA level (c), ALA level (d), EPA level (e) and the percentage of EPA (TFA \%) (f) were determined in the control strain (WT M. alpina) and transformants (including DsDsFADS6-9, Ds-DsFADS6-4, Ds-TpFADS6-8, and Ds-TpFADS6-10). Each alga was cultured in Ben-Amotz medium by supplementation with 500 mg/L $\mathrm{MI}$, glucose $/ \mathrm{KNO}_{3}$ and $12 \% \mathrm{CO}_{2}$ aeration under varying temperature, containing PeSM-free, 1, 2 or $4 \mathrm{~g} / \mathrm{L}$ PeSM as the exogenous substrate

\%) increased. The conversion rate of ALA to EPA in TpFADS6 transformants was greater than that in S. cerevisiae. This may be explained by the optimization of codon usage for TpFADS6 in D. salina, but not in S. cerevisiae. It may be also explained by differences in the levels of LA and ALA in D. salina. TpFADS6 had a higher conversion rate for ALA in $D$. salina (which had higher levels of ALA) than in S. cerevisiae (in which similar levels of both substrates were added).

Many previous studies have shown that auxins play key roles in algal growth $[15,21]$. In this study, the DCWs of WT $D$. salina and the transformants were significantly increased by MI supplementation, which was expected, as $\mathrm{MI}$ is involved in many physiological functions [15, 
22]. MI is safe for use in humans and its cost is relatively low, supporting its use as a growth-promoting agent for large-scale PUFA production in D. salina. Many studies have reported that $\mathrm{CO}_{2}$ fixation rates in microalgae are much greater than those in terrestrial plants [23]. Many microalgae tolerate $\mathrm{CO}_{2}$ levels of up to $12.0 \%$ [24], but algal growth can be affected by high $\mathrm{CO}_{2}$ levels [25]. Our results were consistent with these previous results. At a high $\mathrm{CO}_{2}$ level, the DCW of D. salina may be decreased, owing to the reduced levels of dissolved oxygen in the medium, and lipid biosynthesis in D. salina may increase. Aeration with $\mathrm{CO}_{2}$ in D. salina culture medium not only increases lipid levels but also promotes the conversion of $\mathrm{CO}_{2}$ (inorganic substance) to lipids (organic substance).

Surprisingly, after Ds-TpFADS6 transformants were maintained at $4{ }^{\circ} \mathrm{C}$ for 5 days, their ALA levels increased and LA levels decreased. We reasoned that the activity of the DsFADS15 enzyme was enhanced at the low temperature, promoting the conversion of LA to ALA and increasing the ALA level. These findings are similar to those of Okuda [26], who observed accumulation of EPA in Mortierella alpina at a low temperature (below $15{ }^{\circ} \mathrm{C}$ ). However, FADS15 in M. alpina had high activity for $\mathrm{C} 20$ substrates, whereas DsFADS15 had high activity for $\mathrm{C} 18$ substrates. Ds-TpFADS6 transformants exhibited greater EPA accumulation under varying temperatures than under constant temperatures, suggesting that the LA-ALA-EPA pathway was more active under varying temperatures, and there was less LA flux through the $\omega 6$ pathway. Additional studies are needed to clarify the molecular mechanism of DsFADS15 activity under low temperatures.

Many plant seeds are rich in ALA, such as linseeds, tree peony seeds, sesame seeds, and perilla seeds [2730]; among them, perilla seeds have the highest ALA content [29]. Accordingly, perilla seed oil has applications for both food and medicine. The byproduct of perilla seed oil processing (PeSM) is not widely used. Most PeSM is used as a protein source for animal feed [31]. In addition to ALA, PeSM also contains a high protein content and many other bioactive compounds, making it an inexpensive and undervalued algal material. D. salina is capable of using organic nitrogen for its growth [32, 33], and PeSM is a suitable nitrogen source. The solubility of PeSM is much greater than that of peony seed meal (PSM), which is used for M. alpina growth [34]. However, a high concentration of PeSM can still affect $D$. salina cell growth owing to increased levels of insoluble substances from PeSM in cultures under high concentrations.

EPA is found in a wide variety of marine microalgae. Recently, some remarkable findings in the generation of transgenic microalgae for enhanced EPA production have been reported [35-37]. D. salina is widely used for $\beta$-carotene production [38, 39]. In addition, it accumulates high levels of lipids and triacylglycerides. However, its EPA levels are low, and EPA production by $D$. salina has not been evaluated. In this study, several strategies were used to improve EPA accumulation by $D$. salina. However, investigations of EPA production by microalgae are still in the early stages, and an in-depth understanding of the factors that affect EPA production is still needed. Genetic engineering may be the most efficient means to improve EPA production in microalgae.

\section{Conclusions}

In this study, we identified FADS6 from D. salina. DsFADS6 had a preference for LA as a substrate and TpFADS6 had no substrate preference when expressed in S. cerevisiae. We successfully overexpressed the TpFADS6 gene in $D$. salina; to our knowledge, this represents the first report of EPA bioproduction via the $\omega 3$ pathway in D. salina. EPA production in Ds-TpFADS6 transformants was enhanced to various degrees by $\mathrm{MI}, \mathrm{CO}_{2}$, low temperature, and PeSM supplementation.

\section{Methods \\ Strains and plasmids}

Dunaliella salina (D. salina) was newly isolated from the reef on the beach in the northeast region of China. TpFADS6 gene (GenBank accession AY817155) was synthesized by Shanghai Sunny Biotechnology Co. Ltd; INVSc1 yeast strain (Invitrogen) was used for heterogeneous expression and substrate preference determination. Plasmid pGEM-CAT was used for FADS6 expression in D. salina.

\section{Media and cultural conditions}

D. salina was grown at $26^{\circ} \mathrm{C}$ on Ben-Amotz medium [40] in $12 \mathrm{~h}$ light $(4500 \mathrm{~lx}) / 12 \mathrm{~h}$ dark cycle. SC-U was synthetic minimal defined medium for Saccharomyces cerevisiae [12]. Biomass-enhancing medium was the Ben-Amotz modified medium by supplementation with $500 \mathrm{mg} / \mathrm{L}$ myo-inositol (MI) [15]. Lipid-producing medium was the Ben-Amotz modified medium by aerating with $12 \% \mathrm{CO}_{2}$ level mixed with ambient air [16], and supplementation with $50 \mathrm{mM}$ glucose $(9 \mathrm{~g} / \mathrm{L})$ and $10 \mathrm{mM} \mathrm{KNO} \mathrm{K}_{3}(1 \mathrm{~g} / \mathrm{L})$ which was the result of our preliminary experiment for lipid production in D. salina. PeSM $(1,2$ and $4 \mathrm{~g} / \mathrm{L})$ was added in lipid-producing medium as exogenous substrates for more lipid production, and was prepared by previous procedure for peony seed meal [34].

\section{RNA isolation and gene synthesis}

$1 \mu \mathrm{g}$ RNA of $D$. salina cells was reverse-transcribed with QuantScript RT Kit according to the manufacturer's instruction. The cDNA transcribed was used as 
a template for DsFADS6 amplification with primers. Codon-optimized TpFADS6 gene was synthesized by Biotechnology Co. Ltd and subcloned into the vector pUC57 and transformed into DH5 $\alpha$.

\section{Primer design, PCR amplification, and sequence analysis for DsFADS6 and TpFADS6}

To identify genes encoding DsFADS6, a PCR-based cloning strategy was adopted. According to the available sequences information of FADS6 from Parietochloris incisa, Thalassiosira pseudonana Glossomastix chrysoplasta and Phaeodactylum tricornutum (GenBank accession nos. GU390532, AY817155, AAU11445 and AY082393), two highly degenerate primers (Additional file 1: Table S1) were designed to target sequences corresponding to the heme-binding motif of the cyt b5-like domain and the third His-rich motif in DsFADS6. The amplified product of expected length (DsFADS6 partial sequence, about $700 \mathrm{bp}$ ) was ligated into pMD19-T simple vector and then sequenced. DsFADS6 partial sequence obtained was used to do BLAST search on GenBank (NCBI). Two degenerate primers (Additional file 1: Table S1) were designed to clone the upstream sequence from HPGG and the downstream sequence from QIEHH. Both amplified products were sequenced and located the start codon and the stop codon. After the full length cDNA of DsFADS6 was amplified, it was then ligated into pMD19-T simple vector and sequenced.

For DsFADS6 and TpFADS6 gene amplification, primers were synthesized based on DsFADS6 and TpFADS6 gene sequences. The forward primers were FDs \& FTp and the reverse primers were RDs and RTp (all primers are listed in Additional file 1: Table S1). PCR amplification, expression vector construction and sequencing were based on previous study [12], and recombinant plasmids were designated pYES2/NT C-DsFADS6 and pYES2/NT C-TpFADS6.

Yeast transformation, heterologous expression in S. cerevisiae, and determination of substrate preference for DsFADS6 and TpFADS6

pYES2/NT C-DsFADS6 and pYES2/NT C-TpFADS6 were transformed into $S$. cerevisiae using the lithium acetate transformation method [41]. The selection procedure,
SDS-PAGE gel and Western blotting for expression of DsFADS6 and TpFADS6 were analyzed as described previously [12]. After induction, cultures were supplemented with substrates (as described previously [12]). Substrate concentration experiment, lipids extraction and determination were also analyzed as described previously [34].

\section{Plasmid construction in D. salina}

DsFADS6 and TpFADS6 genes were amplified with pGEM-FDs/pGEM-RDs and pGEM-FTp/pGEM-RTp primers, respectively, as shown in Additional file 1: Table S1. To construct an expression vector pGEM-CAT, the Ubiquitin- $\Omega$ (Ubil- $\Omega$ ) promoter and nos terminator was amplified and cloned into the pGEM control vector to generate the expression vector pGEM $\Omega$-CAT containing the Ubil- $\Omega$ promoter, nos terminator and CAT gene. DsFADS6 was digested and subcloned into Hind III and Xho I sites to generate a plasmid designated pGEM $\Omega$-CAT-DsFADS6 while TpFADS6 was subcloned into EcoR I and Xho I sites to generate a plasmid designated pGEM $\Omega$-CAT-TpFADS6. The expression plasmids (pGEM $\Omega$-CAT-DsFADS6 and pGEM $\Omega$-CAT-TpFADS6) were transferred into $D$. salina cells according to the method described by Wang et al. [42].

\section{CAT assays and RT-qPCR analysis}

The pre-procedure of CAT assays for stably $D$. salina transformants was followed by Wang's description [42]. RT-qPCR analysis procedure were followed as previously described [34]. The transcript levels were calculated using the $2^{-\Delta \Delta C t}$ method [43].

\section{PUFAs production for $D$. salina transformants}

$D$. salina transformant cells in the liquid medium were grown for 30 days. The $D$. salina lysates obtained above were also used to determine dry cell weight (DCW) and fatty acid profiles in triplicates. FA profiles were investigated by using Gas Chromatography (GC) analysis as described previously [34]. D. salina transformant with the highest EPA production and the control were grown in biomass-enhancing medium for biomass enhancement, and grown in the lipid-producing medium for lipid enhancement. Finally, they were separately grown in EPA-enhancing medium for more EPA production. 


\section{Additional files}

Additional file 1: Table S1. Primers used in this study. Table S2. The composition of PeSM (\%).

Additional file 2: Figure S1. Schematic illustration and transformation of expression vectors containing DsFADS6 gene and TpFADS6 gene, respectively. (a) Construction of vector PGEM-CAT-DSFADS6; (b) construction of vector pGEM-CAT-TpFADS6. Ten pGEM-CAT-DsFADS6 transformants and ten pGEM-CAT-TPFADS6 transformants were randomly picked out with PCR amplification for checking transformation of vector pGEM-CAT-DsFADS6 (c) and pGEM-CAT-TPFADS6 (d) into D. salina cells. M Marker; 1: positive control: DsFADS6 gene (c) and TpFADS6 gene (d); 2-11: each transformant.

Additional file 3: Figure S2. Gas chromatogram of fatty acids in WT D. salina with free-supplementation at $26{ }^{\circ} \mathrm{C}$ in Ben-Amotz medium (a) and Ds-TpFADS6-10 transformant by supplementing with $4 \mathrm{~g} / \mathrm{L}$ perilla seed meal, $500 \mathrm{mg} / \mathrm{L} \mathrm{Ml}$ and $12 \% \mathrm{CO}_{2}$ aeration with $50 \mathrm{mM}$ glucose $/ 10 \mathrm{mM}$ $\mathrm{KNO}_{3}$ in varying temperature in Ben-Amotz medium (b).

\section{Authors' contributions}

HS designed and carried out this work, and drafted the manuscript. XL analyzed the data and helped to draft the manuscript. RW supervised the research and helped to draft the manuscript. XY conceived the study and revised the manuscript. All authors read and approved the final manuscript.

\section{Acknowledgements}

Not applicable.

\section{Competing interests}

The authors declare that they have no competing interests.

\section{Availability of data and materials}

There was no new software, database or application/tool used in our manuscript.

\section{Consent for publication}

All authors have read and approved this version of the article, and consented for publication.

\section{Ethics approval and consent to participate}

Not applicable.

\section{Funding}

This study was supported by the National Natural Science Foundation of China (No. 31471713) and Program for Liaoning Excellent Talents in University (LR2015059).

\section{Publisher's Note}

Springer Nature remains neutral with regard to jurisdictional claims in published maps and institutional affiliations.

Received: 7 April 2017 Accepted: 2 January 2018

Published online: 13 January 2018

\section{References}

1. Voigt RG, Jensen CL, Fraley JK, Rozelle JC, Brown FR, Heird WC. Relationship between omega 3 long-chain polyunsaturated fatty acid status during early infancy and neurodevelopmental status at 1 year of age. J Hum Nutr Diet. 2002;15:111-20.

2. Calder PC. n-3 polyunsaturated fatty acids and inflammation: from molecular biology to the clinic. Lipids. 2003;38:343-52.

3. Meyer BJ, Mann NJ, Lewis JL, Milligan GC, Sinclair AJ, Howe PRC. Dietary intakes and food sources of omega- 6 and omega-3 polyunsaturated fatty acids. Lipids. 2003;38:391-8.
4. Parker G. Omega-3 fatty acids and mood disorders-Reply. Am J Psychiatry. 2006;163:2018-9.

5. Nagel G, Nieters A, Becker N, Linseisen J. The influence of the dietary intake of fatty acids and antioxidants on hay fever in adults. Allergy. 2003;58:1277-84

6. Simopoulos AP. Omega-3 fatty acids in inflammation and autoimmune diseases. J Am Coll Nutr. 2002:21:495-505.

7. Kremer JM, Lawrence DA, Petrillo GF, Litts LL, Mullaly PM, Rynes RI, Stocker RP, Parhami N, Greenstein NS, Fuchs BR, et al. Effects of high-dose fish-oil on rheumatoid-arthritis after stopping nonsteroidal antiinflammatory drugs - clinical and immune correlates. Arthritis Rheum. 1995;38:1107-14

8. Ueshima H, Stamler J, Elliott P, Chan Q, Brown IJ, Carnethon MR, Daviglus ML, He K, Moag-Stahlberg A, Rodriguez BL, et al. Food omega-3 fatty acid intake of individuals (total, linolenic acid, long-chain) and their blood pressure INTERMAP study. Hypertension. 2007;50:313-9.

9. Schaefer EJ, Bongard V, Beiser AS, Lamon-Fava S, Robins SJ, Au R, Tucker $\mathrm{KL}$, Kyle DJ, Wilson PWF, Wolf PA. Plasma phosphatidylcholine docosahexaenoic acid content and risk of dementia and Alzheimer disease-The Framingham heart study. Arch Neurol. 2006:63:1545-50.

10. Garcia SM, Rosenberg AA. Food security and marine capture fisheries: characteristics, trends, drivers and future perspectives. Philos Trans R Soc B-Biol Sci. 2010;365:2869-80.

11. Goold H, Beisson F, Peltier G, Li-Beisson Y. Microalgal lipid droplets: composition, diversity, biogenesis and functions. Plant Cell Rep. 2015;34:545-55.

12. Shi H, Chen H, Gu Z, Song Y, Zhang H, Chen W, Chen YQ. Molecular mechanism of substrate specificity for delta 6 desaturase from Mortierella alpina and Micromonas pusilla. J Lipid Res. 2015:56:2309-21.

13. Tonon T, Sayanova O, Michaelson LV, Qing R, Harvey D, Larson TR, Li Y, Napier JA, Graham IA. Fatty acid desaturases from the microalga Thalassiosira pseudonana. FEBS J. 2005;272:3401-12.

14. Stevenson JM, Perera IY, Heilmann I, Persson S, Boss WF. Inositol signaling and plant growth. Trends Plant Sci. 2000;5:252-8.

15. Cho K, Kim K-N, Lim N-L, Kim M-S, Ha J-C, Shin HH, Kim M-K, Roh SW, Kim D, Oda T. Enhanced biomass and lipid production by supplement of myo-inositol with oceanic microalga Dunaliella salina. Biomass Bioenerg. 2015;72:1-7.

16. El Baky HHA, El-Baroty GS, Bouaid A. Lipid induction in Dunaliella salina culture aerated with various levels $\mathrm{CO} 2$ and its biodiesel production. J Aquac Res Dev. 2014;5:1.

17. Zaslavskaia LA, Lippmeier JC, Shih C, Ehrhardt D, Grossman AR, Apt KE. Trophic conversion of an obligate photoautotrophic organism through metabolic engineering. Science. 2001:292:2073-5.

18. Liu J. Optimisation of biomass and lipid production by adjusting the interspecific competition mode of Dunaliella salina and Nannochloropsis gaditana in mixed culture. J Appl Phycol. 2014;26:163-71.

19. Hamilton ML, Haslam RP, Napier JA, Sayanova O. Metabolic engineering of Phaeodactylum tricornutum for the enhanced accumulation of omega-3 long chain polyunsaturated fatty acids. Metab Eng. 2014;22:3-9.

20. Cho K, Lee C-H, Ko K, Lee Y-J, Kim K-N, Kim M-K, Chung Y-H, Kim D, Yeo I-K, Oda T. Use of phenol-induced oxidative stress acclimation to stimulate cell growth and biodiesel production by the oceanic microalga Dunaliella salina. Algal Research-Biomass Biofuels and Bioproducts. 2016;17:61-6.

21. Lau SSN, Bock R, Jürgens $G$, De Smet I. Auxin signaling in algal lineages: fact or myth? Trends Plant Sci. 2009;14:182-8.

22. JM Stevenson IP, Heilmann I, Persson S. Inositol signaling and plant growth. Trends Plant Sci. 2000;5:252-8.

23. Ho SHCWM, Chang JS. Scenedesmus obliquus CNW-N as a potential candidate for CO 2 mitigation and biodiesel production. Biores Technol. 2010;101:8725-30.

24. Chiu S-Y, Kao C-Y, Tsai M-T, Ong S-C, Chen C-H, Lin C-S. Lipid accumulation and $\mathrm{CO} 2$ utilization of Nannochloropsis oculata in response to $\mathrm{CO} 2$ aeration. Biores Technol. 2009;100:833-8.

25. Ota M, Kato Y, Watanabe H, Watanabe M, Sato Y, Smith RL, Inomata H. Fatty acid production from a highly $\mathrm{CO} 2$ tolerant alga, Chlorocuccum littorale, in the presence of inorganic carbon and nitrate. Biores Technol. 2009:100:5237-42.

26. Okuda T, Ando A, Negoro H, Muratsubaki T, Kikukawa H, Sakamoto T, Sakuradani E, Shimizu S, Ogawa J. Eicosapentaenoic acid (EPA) production by an oleaginous fungus Mortierella alpina expressing heterologous 
the $\Delta 17$-desaturase gene under ordinary temperature. Eur J Lipid Sci Technol. 2015;117(12):1919-27.

27. Petrovic M, Gacic M, Karacic V, Gottstein Z, Mazija H, Medic H. Enrichment of eggs in $\mathrm{n}-3$ polyunsaturated fatty acids by feeding hens with different amount of linseed oil in diet. Food Chem. 2012;135:1563-8.

28. Sugasini D, Lokesh BR. Uptake of alpha-linolenic acid and its conversion to long chain omega-3 fatty acids in rats fed microemulsions of linseed oil. Lipids. 2012;47:1155-67.

29. Igarashi M, Miyazaki Y. A review on bioactivities of Perilla: progress in research on the functions of Perilla as medicine and food. Evid Based Complement Altern Med. 2013;2013:925342. https://doi. org/10.1155/2013/925342

30. Pathak N, Rai AK, Kumari R, Bhat KV. Value addition in sesame: a perspective on bioactive components for enhancing utility and profitability. Pharmacogn Rev. 2014;8:147-55.

31. Tang WSB, Zhao Y. Preparative separation and purification of rosmarinic acid from perilla seed meal via combined column chromatography. J Chromatogr B. 2014:947:41-8.

32. Berg G, Glibert P, Lomas M, Burford M. Organic nitrogen uptake and growth by the chrysophyte Aureococcus anophagefferens during a brown tide event. Mar Biol. 1997;129:377-87.

33. Lomas MW, Glibert PM, Clougherty DA, Huber DR, Jones J, Alexander J, Haramoto E. Elevated organic nutrient ratios associated with brown tide algal blooms of Aureococcus anophagefferens (Pelagophyceae). J Plankton Res. 2001;23:1339-44.

34. Shi H, Chen H, Gu Z, Zhang H, Chen W, Chen YQ. Application of a delta-6 desaturase with a-linolenic acid preference on eicosapentaenoic acid production in Mortierella alpina. Microb Cell Fact. 2016;15:117.

35. Zaslavskaia LA, Lippmeier JC, Shih C, Ehrhardt D, Grossman AR, Apt KE. Trophic conversion of an obligate photoautotrophic organism through metabolic engineering. Science. 2001;292:2073-5.
36. Wen ZYCF. Heterotrophic production of eicosapentaenoic acid by microalgae. Biotechnol Adv. 2003;21:273-94.

37. Wen ZYCF. A perfusion-cell bleeding culture strategy for enhancing the productivity of eicosapentaenoic acid by Nitzschia laevis. Appl Microbiol Biotechnol. 2001;57:316-22.

38. Fu W, Guððmundsson Ó, Paglia G, Herjólfsson G, Andrésson ÓS, Palsson $B \varnothing$, Brynjólfsson S. Enhancement of carotenoid biosynthesis in the green microalga Dunaliella salina with light-emitting diodes and adaptive laboratory evolution. Appl Microbiol Biotechnol. 2013:97:2395-403.

39. Lamers PP, van de Laak CC, Kaasenbrood PS, Lorier J, Janssen M, De Vos RC, Bino RJ, Wijffels RH. Carotenoid and fatty acid metabolism in lightstressed Dunaliella salina. Biotechnol Bioeng. 2010;106:638-48.

40. Koberg M, Cohen M, Ben-Amotz A, Gedanken A. Bio-diesel production directly from the microalgae biomass of Nannochloropsis by microwave and ultrasound radiation. Biores Technol. 2011;102:4265-9.

41. Gietz D, Stjean A, Woods RA, Schiestl RH. Improved method for high-efficiency transformation of intact yeast-cells. Nucleic Acids Res. 1992;20:1425.

42. Wang T, Xue L, Hou W, Yang B, Chai Y, Ji X, Wang Y. Increased expression of transgene in stably transformed cells of Dunaliella salina by matrix attachment regions. Appl Microbiol Biotechnol. 2007;76:651-7.

43. Wang $\mathrm{H}$, Yang $B$, Hao G, Feng $Y$, Chen $H$, Feng L, Zhao J, Zhang $H$, Chen $Y Q$, Wang L, Chen W. Biochemical characterization of the tetrahydrobiopterin synthesis pathway in the oleaginous fungus Mortierella alpina. Microbiology-Sgm. 2011;157:3059-70.

44. Venegas-Caleron M, Sayanova O, Napier JA. An alternative to fish oils: metabolic engineering of oil-seed crops to produce omega-3 long chain polyunsaturated fatty acids. Prog Lipid Res. 2010;49:108-19.

\section{Submit your next manuscript to BioMed Central and we will help you at every step:}

- We accept pre-submission inquiries

- Our selector tool helps you to find the most relevant journal

- We provide round the clock customer support

- Convenient online submission

- Thorough peer review

- Inclusion in PubMed and all major indexing services

- Maximum visibility for your research

Submit your manuscript at www.biomedcentral.com/submit
O Biomed Central 